\title{
Minimally invasive parathyroidectomy in patients with previous neck surgery
}

\author{
Spiridon Dimas, ${ }^{1}$ Spiridon Michas, ${ }^{1}$ Ioannis Christakis, ${ }^{1}$ \\ Christos Augoustis, ${ }^{2}$ Maria Alevizaki ${ }^{3}$
}

Department of ${ }^{1}$ Surgery and ${ }^{2}$ Endocrinology "Polykliniki" General Hospital, Athens, Endocrine Unit, Dept of Medical Therapeutics, Athens University School of Medicine ${ }^{3}$

\begin{abstract}
OBJECTIVE: Previous neck surgery (PNS) in patients with primary hyperparathyroidism (PHP) is considered as a contraindication for minimally invasive parathyroidectomy (MIP). The purpose of our study was to determine the effectiveness of MIP in such patients. DESIGN: From January 2003 to June 2011, 380 patients with PHP were treated in our department; 42 had had previous neck surgery. Twenty-seven (27/42) were selected to have MIP; the remaining 15 patients had traditional neck explorations. Selection criteria for MIP were unilateral single or two gland disease localized preoperatively with at least two imaging techniques and patient's informed consent. Imaging studies included high resolution neck ultrasound and sestamibi scan in the majority, and CT scan, selective venous sampling and MRI in seven patients. The type of operation done included unilateral approach under local anesthesia (UALA) (22 cases) with one conversion to general anesthesia and minimally invasive parathyroidectomy under general anesthesia (MIPG) (5 cases). RESULTS: Twenty-six of the 27 patients became normocalcemic after the operation. The patient with persistent hypercalcemia underwent successful parathyroidectomy 8 months later via mesothoracoscopy, since the parathyroid gland was localized correctly but was beyond access via neck. A single adenoma was found in 21 cases and hyperplasia in six. There were no conversions to traditional exploration and no postoperative complications. Mean duration of the procedure and length of stay were similar to MIP in patients without PNS. Mean follow-up of 40 months (4-89 months) did not reveal any recurrence. CONCLUSION: These results illustrate that MIP is a valuable option in selected patients with PHP and PNS associated with no morbidity (0\%), high biochemical cure rate (96.3\% in this series) and rapid recovery, while it also substantially lowers the cost of the procedure. Preoperative localization with two or more agreeing imaging techniques eliminates the need for intraoperative sestamibi or qPTH test.
\end{abstract}

Key words: Minimally invasive surgery, Parathyroidectomy, Primary hyperparathyroidism 


\section{INTRODUCTION}

Minimally invasive parathyroidectomy (MIP) has been accepted as the procedure of choice for the majority of patients with primary hyperparathyroidism (PHP) and single gland disease and seems to have replaced the gold standard of bilateral neck exploration. The success results of such minimally invasive procedures are reported to be over $96 \%$ in the literature. ${ }^{1,2}$ Previous neck surgery (PNS), especially total thyroidectomy, in patients with sporadic PHP has been considered by many authors as a contraindication for MIP. This belief has been challenged over the last 15 years thanks to a group of surgeons who pioneered the use of minimally invasive parathyroidectomy in these patients and reported a very high success rate. However, it is well known that such results may vary between centers. Thus, the purpose of this retrospective study was to evaluate the feasibility of MIP in patients with previous neck surgery and to establish the success rates.

\section{PATIENTS AND METHODS}

From January 2003 to June 2011, 380 cases with PHP were treated in our department. After detailed history, 42 of the 380 patients $(11 \%)$ were found to have had previous neck surgery (most cases were classical total thyroidectomies). Minimally invasive parathyroidectomy was performed only when there was unilateral single or two gland disease and the adenoma(s) was preoperatively localized with two concordant imaging techniques, one of which necessarily was sestamibi scan. Whenever those criteria were not met, patients were scheduled for bilateral neck exploration. Twenty-seven out of the 42 cases $(64.3 \%)$ fulfilled the selection criteria; the remaining 15 patients failed to fulfill both of the selection criteria and underwent bilateral neck exploration.

All patients had preoperative localization with ${ }^{99 \mathrm{~m}}$ Tc-labelled sestamibi scan imaging and high resolution ultrasonography of the neck. CT scan, PTH with selective venous sampling and MRI was performed in seven patients.

Previous endocrine neck operations had been performed in 23 patients of the first group (total or subtotal thyroidectomy) before the diagnosis of PHP, except for one patient who had a 2-stage planned unilateral approach under local anesthesia (UALA) for parathyroid hyperplasia, and a further two patients who had MEN IIA syndrome and had undergone insufficient previous parathyroidectomy. Four patients had a previous operation and/or radiation for other reasons. The decision to perform UALA or MIP under general anesthesia (MIPG) in these patients, just as in the non-operated group, was based on experience.

All of the patients with PNS had a preoperative voice laryngoscopy to exclude unsuspected previous damage to the recurrent laryngeal nerves. Twenty-two of the 27 patients underwent UALA and 5 MIPG. Depending on the patient's compliance and comfort, the anesthesiologist used light sedation with small doses of intravenous propofol (18 cases) when needed. In the event of persistent patient's discomfort, despite the sedation, the procedure was converted to MIPG (1 patient).

A 2 to $2.5 \mathrm{~cm}$ transverse incision was made in a skin crease directly over the localized parathyroid gland along the front border of the sternocleidomastoid muscle. The strap muscles were divided by sharp dissection with the scissors until the neurovascular cervical bundle was encountered. The carotid artery and the lateral margin of the thyroid gland remnant was recognized when present and the plane of dissection continued with a medial direction without any hemorrhage until the parathyroid adenoma(s) were identified. It is important to stress that in the majority of cases the dissection and removal of the glands were surprisingly easy without any hemorrhage. A silicon negative pressure drain was placed only in cases of difficult dissection. No gamma probe, qPTH assays or frozen-section analysis were performed.

All patients were considered for discharge on the $1^{\text {st }}$ postoperative day after the morning measurement of blood calcium levels. Calcium and vitamin $\mathrm{D}_{3}$ tablets were given on discharge in cases in which there was a high drop of blood calcium levels. In this case, patients returned for a second measurement on the $3^{\text {rd }}$ or $5^{\text {th }}$ postoperative day. The duration of the operation, the length of stay, the conversions and complications rates were recorded prospectively. All patients were seen in the outpatient clinic on the $5^{\text {th }}$ postoperative day in order to remove the steri-strips 
bands and undergo assessment for complications. Further follow-up was at 5-12 months.

\section{RESULTS}

Over a period of 9.5 years, 380 patients underwent treatment for primary HPT and 42 of them had a previous neck surgery. Only 27 out of the 42 patients with PNS fulfilled the criteria for MIP. In this group the sex ratio $(\mathrm{M} / \mathrm{F})$ was $6 / 21$ and the mean age 65.7 (range 35-83 years).

In 20 out of the 27 patients which were selected, the sestamibi scanning was concordant with the neck ultrasound and no further imaging was performed. In seven patients the two methods that were mentioned were non-concordant and further imaging was performed comprising Computer Tomography or
Magnetic Resonance Imaging of the neck (3 cases) and selective venous sampling (4 cases).

The decision on the type of anesthesia to be used in this group, local vs. general, was based on the patient's individual preferences. Out of a total of 27 patients, 22 patients had UALA and 5 patients had MIPG. In only one case the operation was converted from UALA to MIPG because of patient's anxiety and discomfort (Figure 1). Basic characteristics of the study population are shown in Table 1.

A single parathyroid adenoma was identified in 21 patients and there were six cases of parathyroid hyperplasia. The location of the excised adenomas was in 11 cases right inferiorly, in 7 cases left inferiorly, in 2 cases right superiorly, in 1 case left superiorly and in 5 cases at the upper mediastinum or thymus gland.

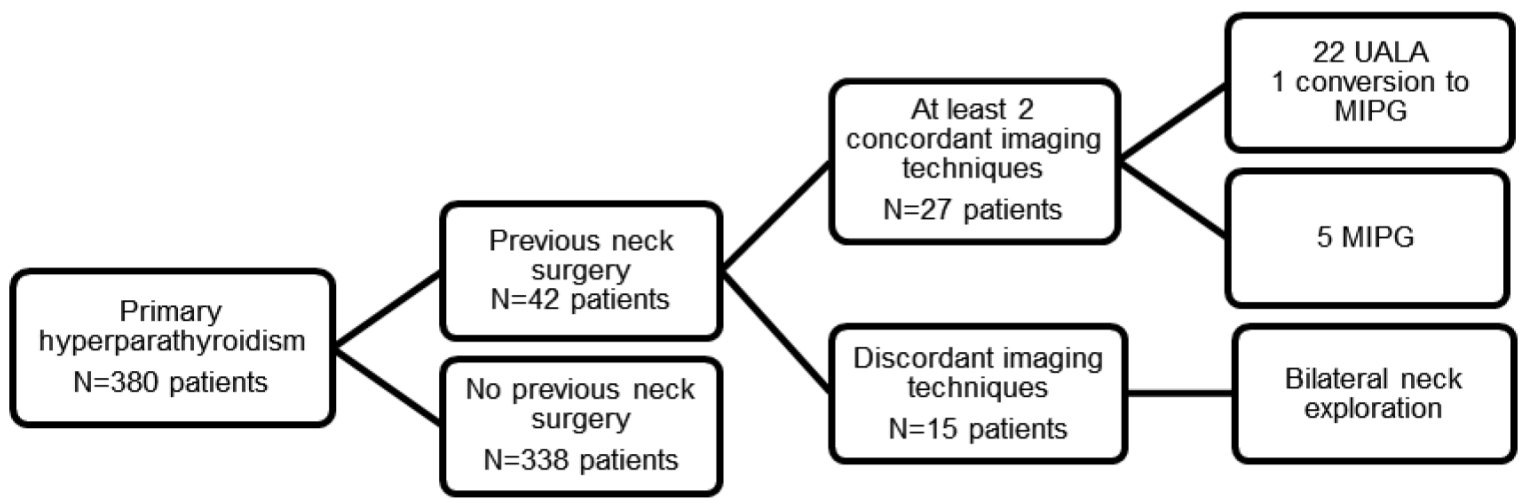

Figure 1. Flow chart of the selection process for patients with minimally invasive parathyroidectomy (MIP). UALA: unilateral approach under local anesthesia; MIPG: MIP-under general anesthesia.

Table 1. Basic characteristics of the study population

\begin{tabular}{|c|c|c|c|c|}
\hline \multicolumn{3}{|c|}{ Type of previous neck surgery in patients selected for MIP } & \multicolumn{2}{|c|}{ Number of patients } \\
\hline \multicolumn{3}{|c|}{ Total or near total thyroidectomy } & \multicolumn{2}{|c|}{20} \\
\hline \multicolumn{3}{|c|}{ Total thyroidectomy and selective parathyroidectomy for MEN IIa } & \multicolumn{2}{|c|}{2} \\
\hline \multicolumn{3}{|l|}{ Thyroidectomy and I131 } & \multicolumn{2}{|c|}{2} \\
\hline \multicolumn{3}{|c|}{ Previous parathyroidectomy } & \multicolumn{2}{|c|}{1} \\
\hline \multicolumn{3}{|c|}{ Highly positioned sternotomy } & \multicolumn{2}{|c|}{1} \\
\hline \multicolumn{3}{|c|}{ Tuberculosis neck multiple abscesses } & \multicolumn{2}{|c|}{1} \\
\hline Mean serum values & Pre-op mean & Pre-op range & Follow-up mean & Follow-up range \\
\hline Total Calcium & $11.2 \mathrm{mg} / \mathrm{dl}$ & $10.9-12.4 \mathrm{mg} / \mathrm{dl}$ & $9.2 \mathrm{mg} / \mathrm{dl}$ & $8.7-10.1 \mathrm{mg} / \mathrm{dl}$ \\
\hline $\mathrm{Ca}++$ & $1.35 \mathrm{mmol} / 1$ & $1.31-1.51 \mathrm{mmol} / \mathrm{l}$ & $1.21 \mathrm{mmol} / \mathrm{l}$ & $1.15-1.27 \mathrm{mmol} / \mathrm{l}$ \\
\hline PTH & $122 \mathrm{pg} / \mathrm{ml}$ & $81-185 \mathrm{pg} / \mathrm{ml}$ & $58 \mathrm{pg} / \mathrm{ml}$ & $42-68 \mathrm{pg} / \mathrm{ml}$ \\
\hline
\end{tabular}

MIP: minimally invasive parathyroidectomy; pre-op: preoperative. 
In one patient who was diagnosed preoperatively with hyperplasia, the decision was made to perform a 2-stage UALA for the first time in our Clinic. In one of the patients of the UALA group, although the parathyroid adenoma was successfully localized preoperatively with two imaging techniques, it was impossible to access it via the neck due to the patient's hunchback and relatively deep localization into the superior mediastinum. Eight months later the patient had a mesothoracoscopy and the parathyroid adenoma was successfully removed.

The mean operating time was 38 minutes (2545 minutes) and it was comparable to the group of patients with MIP without PNS. No surgical complications (nerve damage, hemorrhage, trauma or infection) or persistent hypoparathyroidism occurred. The mean hospital stay was approximately 20 hours (19-23 hours). The patients were discharged on the $1^{\text {st }}$ postoperative day after the morning measurement of blood calcium levels. Twenty-six of the 27 patients became normocalcemic after the operation, and 19 of them required vit. $\mathrm{D}_{3}$ and calcium supplementation for 5 to 20 days. The mean follow-up was 40 months (4-89 months) with no losses to follow-up observed. Until now there have been no reported cases of recurrent HPT or other complications in these patients.

\section{DISCUSSION}

Surgical treatment of PHP has undergone a radical change during the last 15 years and MIP has gained worldwide acceptance, replacing the gold standard of bilateral neck exploration in patients with a presumed solitary parathyroid adenoma, which in fact concerns $80 \%$ of patients with PHP..$^{1-4}$ Better localization techniques and familiarization with minimally invasive surgery have defined MIP as a safe and effective procedure with excellent cure rates when conducted in experienced hands ( $>96 \%)$, similar to those for traditional bilateral neck exploration. ${ }^{4,5}$

MIP is a general term that includes mini incision focusing procedures under local or general anesthesia, video-assisted and endoscopic parathyroidectomy with or without intraoperative gamma probe or IOPTHA. The mini incision focused approach is the current method of choice for the majority of members of the International Association of Endocrine Surgeons. This procedure is minimal not only due to a small incision but also because it involves minimal tissue dissection, enables the operation under local anesthesia an outpatient setting, lowers hospital stay and cost, lowers the incidence of hypocalcemia compared with standard bilateral exploration and results in a smaller scar and less postoperative pain. Its only major disadvantage involves the risk of missing possible multiglandular disease or a second adenoma., ${ }^{2,5,6}$

The success of MIP depends on the preoperative accurate visualization of the diseased parathyroid gland. ${ }^{7}$ The most common preoperative localization techniques are the sestamibi scan and the high resolution ultrasonography of the neck with a high sensitivity and specificity reaching 80 to $90 \%$. The sensitivity increases to values as high as $95-97 \%$, when both of these techniques are concordant. ${ }^{5,8,9}$ Many centers nowadays perform MIP with a $97-98 \%$ success rate without using any intraoperative adjuncts., $3,5,9$ Traditional use of perioperative or intraoperative gamma probe or IOPTHA to confirm the adequacy of resection have not increased the success rate for single gland disease when sestamibi and ultrasound were concordant, even in high volume endocrine surgery centers., ${ }^{3,-12}$ In our study, the CT and MRI scan have been successful in localizing the diseased parathyroid in only three cases, in both of which a giant parathyroid was found.

In the case of non-concordant preoperative localization studies, the use of techniques such as gamma probe or IOPTHA is recommended because the prevalence of multiglandular disease in these cases approaches $17 \%$. Similarly, the use of IOPTHA or gamma probe is recommended for patients undergoing selective parathyroidectomy after a single localization study.,12,13

PNS in patients with sporadic PHP has been considered as a contraindication for MIP, as mentioned earlier. The first attempts at performing MIP in a previously operated neck date back to 1997 when Rotstein and Norman reported success rates of $93 \%$ and $100 \%$ with the use of gamma probe or IOPTHA. ${ }^{14,15}$ It should however be emphasized that the role of quick iPTH assay is still controversial for cost reasons, especially when two concordant results of preoperative imaging tests are available, although 
it is noteworthy that up to $5 \%$ of patients can still benefit. ${ }^{16,17}$

The term PNS or reoperative neck can be divided into two groups. The first group concerns cases with non-parathyroid previous operation (thyroidectomy or other neck procedures) and the second concerns patients with previous parathyroidectomy; thus this means that the second group relates to persistent or recurrent PHP. It is important to stress that persistent or recurrent PHP is indeed a special entity. In these cases, several preoperative localization studies are needed for accurate localization and techniques such as intraoperative gamma probe and IOPTHA can prove valuable. ${ }^{18}$ On the other hand, for cases with non-parathyroid previous neck operation, MIP can be successfully performed when two preoperative localization studies are concordant because the incidence of single adenoma remains near $80 \%$, the same as in PHP in a nonoperated neck.

Based on our results we would suggest that MIP could become the procedure of choice even in patients with previous neck surgery, provided there is enough experience. We would thus certainly agree with Barczynski M. who points out: "it should be stressed that MIP can be recommended only for surgeons with the appropriate experience in conventional parathyroid surgery (bilateral neck exploration). It is not the operation for 'beginners', as there are many potential entrapments which can be encountered during this, easy at first sight, operation. Experience and sound clinical judgment cannot be replaced by any novel technological adjunct". ${ }^{3}$

\section{CONCLUSION}

These results illustrate that MIP using the lateral approach is a valuable option in selected patients with PHP and PNS, even in cases of recurrent or persistent PHP. Localization with two or more agreeing imaging techniques could eliminate the need for intraoperative sestamibi or qPTH test. This procedure in our series was associated with high biochemical cure rate $(96.3 \%)$, rapid recovery and zero morbidity and enabled substantial lowering of the procedure cost.

\section{REFERENCES}

1. Inabnet WB, Fulla Y, Richard B, Bonnichon P, Icard
P, Chapuis Y, 1999 Unilateral neck exploration under local anesthesia: the approach of choice for asyptomatic primary hyperparathyroidism. Surgery 126: 1004-1009.

2. Chapuis Y, 2003 New directions in the surgical treatment of primary hyperparathyroidism. Bull Acad Natl Med 187: 493-502.

3. Barczynski M, 2009 Minimally invasive parathyroidectomy without intraoperative parathyroid hormone monitoring: When and why? J Postgrad Med 55: 239240.

4. Pang T, Stalberg P, Sidhu S, et al, 2007 Minimally invasive parathyroidectomy using the lateral focused mini-incision technique without intraoperative parathyroid hormone monitoring. Br J Surg 94: 315-319.

5. Haciyanli M, Genc H, Damburaci N, Oruk G, Tutuncuoglu P, Erdogan N, 2009 Minimally invasive focused parathyroidectomy without using intraoperative parathyroid hormone monitoring or gamma probe. J Postgrad Med 55: 242-246.

6. Thakur A, Sebag F, Slotema E, Ippolito G, Taïeb D, Henry JF, 2009 Significance of biochemical parameters in differentiating uniglandular from multiglandular disease and limiting use of intraorerative parathormone assay. World J Surg 33: 1219-1223.

7. Quiros R, Alioto J, Wilhelm S, Ali A, Prinz R, 2004 An algorithm to maximize use of minimally invasive parathyroidectomy. Arch Surg 139: 501-507.

8. Ghaheri BA, Koslin DB, Wood AH, Cohen JL, 2004 Preoperative ultrasound is worthwhile for reoperative parathyroid surgery. Laryngoscope 114: 2168-2171

9. Henry Jean-François, 2010 Reoperation for primary hyperparathyroidism: tips and tricks. Langenbecks Arch Surg 395: 103-109.

10. Gawande AA, Monchik JM, Abbruzzese TA, Iannuccilli JD, Ibrahim SI, Moore FD Jr, 2006 Reassessment of parathyroid hormone monitoring during parathyroidectomy for primary hyperparathyroidism after 2 preoperative localization studies. Arch Surg 141: 381-384.

11. Stalberg P, Sidhu S, Sywak M, Robinson B, Wilkinson M, Delbridge L, 2006 Intraoperative parathyroid hormone measurement during minimally invasive parathyroidectomy: does it "value-add" to decision-making? J Am Coll Surg 203:1-6.

12. Gil-Cardenas A, Gamino A, Reza A, Pantoja JP, Herrera MF, 2007 Is intraoperative parathyroid hormone assay mandatory for the success of targeted parathyroidectomy? J Am Coll Surg 204: 286-290.

13. Mihai R, Palazzo FF, Gleeson FV, Sadler GP, 2007 Minimally invasive parathyroidectomy without intraoperative parathyroid hormone monitoring in patients with primary hyperparathyroidism. Br J Surg 94: 42-47.

14. Rotstein L, Irish J, Gullane P, Keller MA, Sniderman K, 1998 Reoperative parathyroidectomy in the era of localization technology. Head Neck 20: 535-539.

15. Norman J, Denham D, 1998 Minimally invasive radioguided parathyroidectomy in the reoperative neck. 
Surgery 124: 1088-1092.

16. Barczynski M, Golkowski F, Konturek A, et al, 2006 Technetium-99m-sestamibi subtraction scintigraphy vs. ultrasonography combined with a rapid parathyroid hormone assay in parathyroid aspirates in preoperative localization of parathyroid adenomas and in directing surgical approach. Clin Endocrinol (Oxf) 65: 106-113.

17. Barczynski M, Konturek A, Cichon S, HubalewskaDydejczyk A, Golkowski F, Huszno B, 2007 Intraop- erative parathyroid hormone assay improves outcomes of minimally invasive parathyroidectomy mainly in patients with a presumed solitary parathyroid adenoma and missing concordance of preoperative imaging. Clin Endocrinol (Oxf) 66: 878-885.

18. Udelsman R, 2011 Approach to the patient with persistent or recurrent primary hyperparathyroidism. J Clin Endocrinol Metab 96: 2950-2958. 